\title{
Relevance of chromatin features in the progression of esophageal epithelial severe dysplasia
}

\author{
Feng Gao ${ }^{1,2}$, Uta Jütting ${ }^{1, *}$, Karsten Rodenacker ${ }^{1}$, Peter Gais ${ }^{1}$ and Pei-zhong Lin $^{2}$ \\ ${ }^{1}$ GSF-Forschungszentrum für Umwelt und Gesundheit, GmbH, Neuherberg, Institut für Pathologie, \\ D-85764 Oberschleißheim, Germany \\ ${ }^{2}$ Cancer Institute, Institute of Pathology, Chinese Academy of Medical Sciences, Beijing 10021, \\ China
}

\begin{abstract}
Since 1983, a long-term clinical trial of esophageal carcinoma chemoprevention has been conducted in a highrisk area in China. From this study, 25 esophageal severe dysplasia patients without therapy were selected for analysis. After 5-year follow-ups, 14 cases progressed to esophageal carcinoma, while the other 11 cases remained stable. Three Papanicolaou's smears were used for each case, including one from the esophageal cytological examination at the beginning, two from the re-examinations three and five years later respectively. About 100 visually normal intermediate cells were randomly collected per slide by high resolution image analysis. More than 100 features (morphologic, densitometric, textural) were extracted. The classifications were made by means of stepwise linear discriminate analysis at the single cell level as on the specimen level using up to ten features. In all three comparisons of patients with progression and with regression at time of diagnosis, three years after diagnosis and five years later, the correct cell classification rates were about $70 \%$. The subsequent specimen classifications by means of the a posteriori probability (APOP) distribution of the cells in each case led to $80 \%$ correct classification. All selected features reflected the chromatin structure of nuclei. The result demonstrated that the chromatin structures of esophageal epithelial cells in severely dysplasic patients are different between cases with and without progression. These results suggest the possibility of the application of image analysis in the clinical trials to find the dysplasia patients with higher risk of progression, in order to reduce the number of patients for therapy.
\end{abstract}

Keywords: Esophageal severe dysplasia, progression, high resolution image analysis, texture feature, malignancy associated changes (MAC)

\section{Introduction}

Esophageal carcinoma is one of the high-incidence tumors in China. Nearly 15,000 people die of it every year. Next to stomach carcinoma, it is the most important death cause from all malignant tumors. In some areas, which are called high-risk areas, the incidence of esophageal carcinoma reaches $0.2 \%$ per year. Therefore, prevention and therapy of esophageal carcinoma are indispensable in high-risk areas. A series of previous research studies has shown that the development of esophageal carcinoma is closely related to esophageal epithelial dysplasia, and severe dysplasia is a precancerous lesion of

\footnotetext{
*Address of correspondence: Uta Jütting, GSF-Institut für Pathologie, Ingolstädter Landstr. 1, D-85764 Oberschleißheim, Germany. Tel.: +49 893187 2555; Fax: +49 8931873349.
} 
esophagus [18]. Thus, it is both essential and possible to treat esophageal epithelial precancerous lesions, inhibit their malignancy and promote regression to normal, in order to achieve the goal of esophageal carcinoma prevention.

Since 1983, a long-term clinical trial of esophageal carcinoma chemoprevention has been conducted in two high-risk areas of Henan Province, China [16,17]. First, an esophageal cytological examination was undertaken among 9,633 residents of 40-65 years of age. The detected 2,531 cases of esophageal severe dysplasia were stratified-randomly and stringently divided into three groups, according to sex, age and the grade of esophageal epithelial lesions. The patients of the three groups were treated with Anti Tumour B (ATB, a purely Chinese medication), Retinamide and a placebo respectively. A quality control system was set up for drug delivery and cancer case registration. After 3- or 5-year treatment, esophageal cytological examinations were repeated. Both re-examination rates amounted to more than $90 \%$. The results showed that ATB and Retinamide reduced the incidence rate of esophageal carcinoma to about $50 \%$, with significant statistical differences $(p<0.01)$ after 3- or 5-year treatments.

According to the studies mentioned above, only a small proportion ( $2 \%$ or so per year) of esophageal severe dysplasia patients progressed to carcinoma without therapy, while most of the cases remained stable [17]. Therefore, it is important to find a method of selecting the cases among dysplasias having a higher risk of progression. Such a method could be used to reduce the number of patients selected for therapy, thus leading to economical and ethical advantages.

In 1959, the first report of malignancy associated changes (MAC) by Nieburgs [24] described morphological changes in nuclei of blood cells from patients with malignant diseases. Since then, such nuclear changes have been found in various tissues, i.e., buccal mucosa [7,22], lung [28,33], cervix [3,41,42], uterus [26], liver [23] and skin [25]. In recent years, some scientists have been using textural features for MAC measurement obtained from high resolution image analysis to classify malignant and benign cases in buccal mucosa [13], soft tissue tumours [9], nasal mucosa [2,32], liver [6], breast [19,37,43], oral mucosa [27], and bladder [40]. Some scientists are proposing to use MAC for the prognosis of cancer or dysplasia cases in breast [5,14,29,31,38], cervix [10,11,20,31], prostate [21] and lung [15,31]. These results are satisfactory when compared with the clinical data.

Our investigation was carried out to determine if morphology, density and texture features obtained from an image analysis system can be used to predict the outcome on esophageal epithelial dysplasia.

\section{Material and methods}

Twenty-five esophageal severe dysplasia cases were selected for analysis from the control group of the clinical trial mentioned above. Fourteen cases progressed to esophageal squamous carcinoma after 5 years. The other 11 cases remained stable. Three smears were used for each case: one from the first esophageal cytological examination and two from the re-examinations 3 and 5 years later.

Smears were obtained by the balloon instrument according to standard method of esophageal cytological examination. Fixation was made using 95\% ethanol, then standard Papanicolaou's stain was carried out. The diagnosis was made by at least two cytologists. The carcinoma cases were identified by means of X-ray, endoscopy and biopsy. All 14 cancer patients selected in this study were operated on or died as the cause of esophageal carcinoma according to clinical data.

\subsection{Data acquisition}

About 100 visually normal intermediate squamous cells per slide were randomly measured with an Axiomat-microscope (Zeiss, Oberkochen, Germany), equipped with a TV-camera (Bosch, T1VK9B1, 


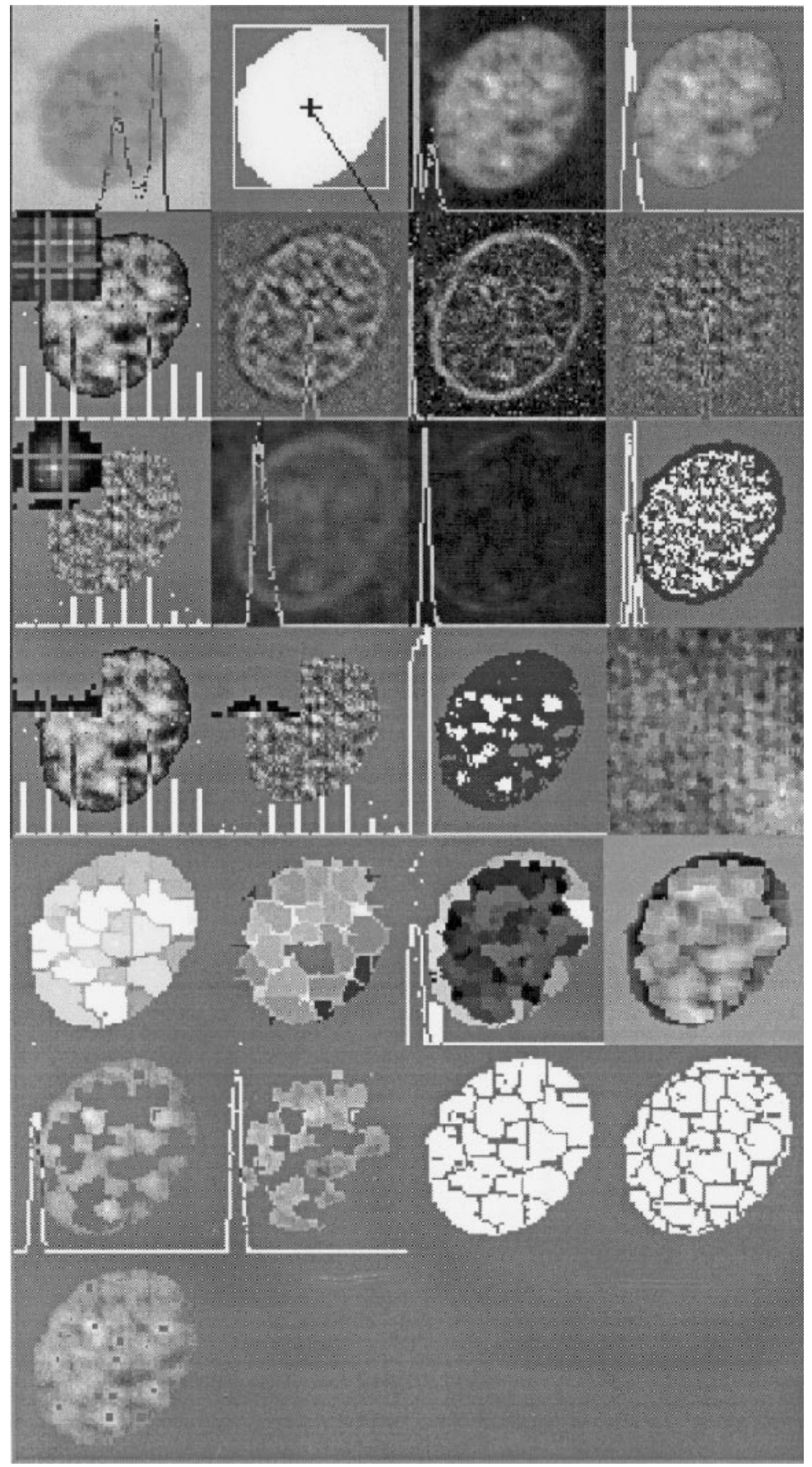

Fig. 1. Output of the feature extraction routine for one cell nucleus. First row: from left to right: transmission, mask, extinction, masked extinction. Second row: co-occurrence matrix on extinction, Laplacian, gradient, flat texture. Third row: co-occurrence on flat texture (NC..), local fractal dimension, local multifractal dimension (MF..), bright/dark particles. Fourth row: runlength on extinction, runlength on flat texture (NR..), Mayall/Young features (hetero), background. Fifth row: skeletonized (upper/lower) extinction image, topological gradient (RG..). Sixth row: bright/dark regions (HL..). 
Stuttgart, Germany, $512 \times 512$ pixel). The cells were scanned in transmission with a $100 \times$ objective (oil immersion, numerical aperture 1.3) using an optical narrow band filter of $548 \mathrm{~nm}$ wavelength. The pixel distance was $0.25 \mu \mathrm{m}$, and the nominal grey value resolution was covered by 256 channels [8]. Processing of digitized images was carried out using a VAX 4000-500 processor (Digital, Maynard, USA) with software written under idl (Interactive Data Language, RSI, Boulder, Colorado, USA). After segmentation of each nucleus by an automatically estimated threshold and a subsequent interactive control, more than 100 features (morphological, densitometrical, textural) were extracted using the extinction or optical density image, which was derived from the transmission image [34]. A shading correction was performed. Densitometric features such as mean, standard deviation, skewness, median, mode, and entropy were calculated from the histogram of the whole nucleus as well as from bright and dark particle regions inside the object that were segmented automatically [34]. For the latter a grey scale skeleton was applied on the extinction image (upper skeleton) and on its inversion (lower skeleton), which delivers the partitions into regions around dark and bright particles. The skeleton is similar to the watershed algorithm. For chromatin distribution features, several transformations were obtained by using linear and non-linear filtering such as Robert's gradient, Laplacean transforms and the above mentioned flat texture image, local fractal and multi-fractal dimensions, topological gradient, the difference of upper and lower skeletons, and statistical features from runlength and co-occurrence matrix $[32,36]$. These textural features are derived from pattern recognition methods. Their biological interpretation has to be derived from the order defined (Fig. 1). Due to nonstoichiometric staining, only those features proven to be independent of staining intensity were used for classification, in order to avoid variance due to preparation and staining [35]. The remaining feature set contained about 60 variables. However the preparation of the smears over the acquisition time differed so much that only specimens collected in the same year could be pooled. But in all three classifications the same subset of features was used. The features are described in the Appendix.

\subsection{Statistics}

The statistical evaluations were made using SAS (SAS Institute, Inc., Cary, NC, USA) and BMDP (Statistical Software Inc., Los Angeles, CA, USA) program packages. All cells from specimens of the same clinical samples were pooled and the classifier was designed by means of a two-class stepwise linear discriminate analysis at the cell level. Of the evaluated feature set only those features were used in the classification steps which were univariate, significant and not highly dependent on staining and preparation methods. Up to 10 features were stepwise selected and were either accepted for the succeeding hold-one-out classification, or the procedure was stopped due to a non-significant $\mathrm{F}$ value. The value for the first selected feature is the univariate one whereas the following F-values are multivariate reflecting the impact of results after using this feature together with the already selected features. For each specimen, the means of the a posteriori probability (APOP) distribution of the corresponding cells was calculated. For the specimen classification, this APOP value and the double standard error of the mean (SEM) were used. A specimen was put into that class with the highest APOP value only if the mean APOP \pm SEM did not cut a threshold (THR) which was set as the border between the two classes. In all cases, the threshold was defined at APOP $=0.5$ that is the half distance between both group means. Cases with $\mathrm{THR} \varepsilon\{\mathrm{APOP} \pm 2 \mathrm{SEM}\}$ were classed as unclear [4]. The significance of the specimen classifications was calculated using contingency tables without defining unclear cases.

All statistical evaluations were done at a $95 \%$ level. 


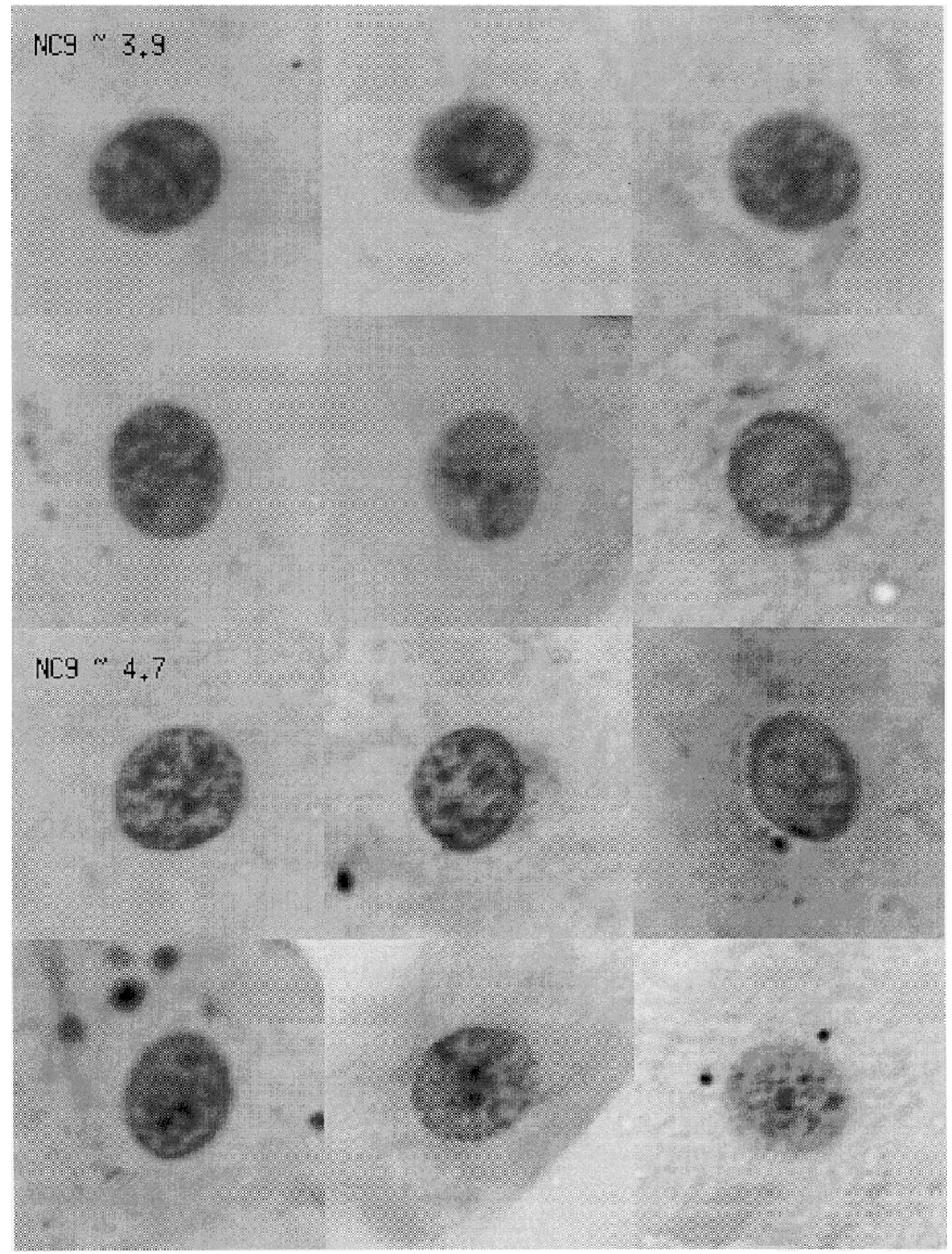

Fig. 2. Nuclei with low (3.9 [A.U.]) and high (4.7 [A.U.]) values of NC9. 


\section{Results}

Due to changes in the preparation and/or staining over the long acquisition time of specimens, the classifications could only be done within the different years (1983, 1987 or 1989, respectively). However, the same subset of 10 chromatin features was used which was more or less independent of staining and preparation changes. No size dependent features were taken into account.

Table 1

Cell and specimen classification results for 1983

\begin{tabular}{lccccc}
\hline & Non-progression & Progression & Total & $\%$ correct & \\
Non-progression & 577 & 256 & 833 & 69.3 & \\
Progression & 299 & 1049 & 1348 & 77.8 & \\
& Non-progression & Progression & Unclear & Total & $\%$ correct \\
Non-progression & 8 & 1 & 1 & 10 & 80.0 \\
Progression & 1 & 11 & 2 & 14 & 78.6 \\
& Non-progression & Progression & Total & $\%$ correct & \\
Non-progression & 8 & 2 & 10 & 80.0 & \\
Progression & 3 & 11 & 14 & 78.6 & \\
\hline
\end{tabular}

$p<0.0001$.

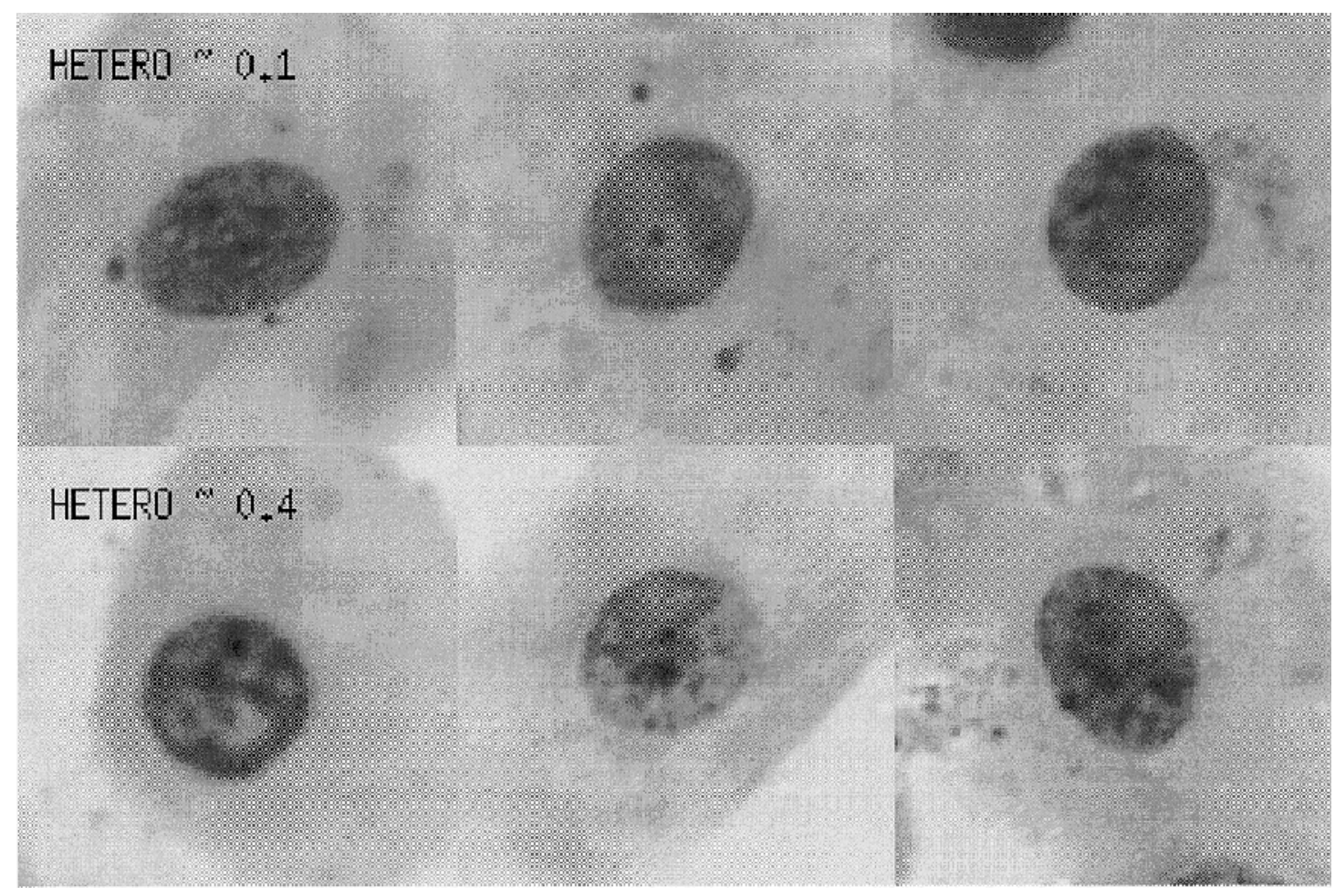

Fig. 3. Nuclei with low (0.1 [A.U.]) and high (0.4 [A.U.]) values of HETERO. 
All cells of specimens belonging to the non-progression or progression group, were pooled and stepwise linear discriminant analysis was applied. The most significant feature was NC9 which calculates the entropy of the median filtered image followed by HETERO and MFRANG (Table 1). Figure 2 shows cells with high (4.7 [A.U.]) and low (3.9 [A.U.]) values of NC9 and Fig. 3 with low and high values for HETERO. In Table 1 the cell and specimen classification results are listed. The correct classification rate of the non-progression group was $69.3 \%$, whereas for the progression group $77.8 \%$ was achieved. The subsequent specimen classification by means of the APOP-value and its standard error of the mean led to 8/10 correct specimen classifications of the non-progression group and 11/14 for the progression group. Three cases were defined as unclear and one case in each group was falsely classified. In Fig. 4 all specimens are ranked according to their mean APOP value. All cases plotted with open symbols were classified as unclear. Without defining the unclear class, these three unclear specimens were misclassified. The result is significant at $p<0.0001$.

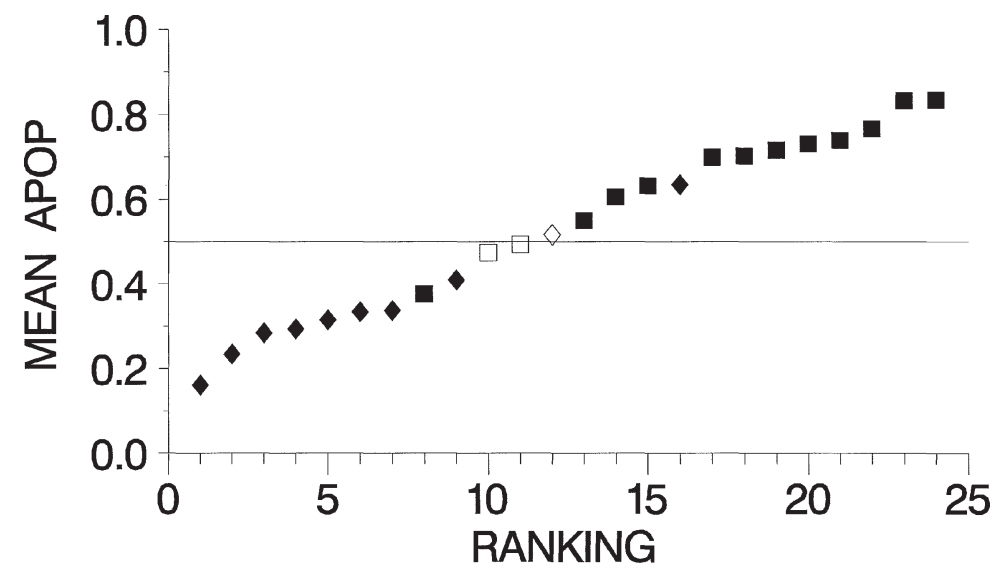

Fig. 4. Cell and specimen classification results of cases sampled in 1983. non-progression, open symbols: unclear decisions.)

Table 2

Cell and specimen classification results for 1987

\begin{tabular}{lccccc}
\hline & Non-progression & Progression & Total & $\%$ correct & \\
Non-progression & 866 & 338 & 1204 & 71.9 & \\
Progression & 396 & 975 & 1371 & 71.1 & \\
& Non-progression & Progression & Unclear & Total & $\%$ correct \\
Non-progression & 8 & 1 & 2 & 11 & 72.7 \\
Progression & 2 & 12 & - & 14 & 85.7 \\
& Non-progression & Progression & Total & $\%$ correct & \\
Non-progression & 8 & 3 & 11 & 72.7 & \\
Progression & 2 & 12 & 14 & 92.9 & \\
\hline$p<0.001$. & & & & &
\end{tabular}

$p<0.001$. 
3.2. Discrimination of patients with non-progression and progression within three years at time of diagnosis (1987)

In this cell classification case the most important features was HLNO, which gives the mean value of chromatin particles in the nuclei extracted in the filtered image after the ricefield transformation, followed by RGM2 (Table 2). Figure 5 shows nuclei with high (21.0 [A.U]) and low (9.0 [A.U.])

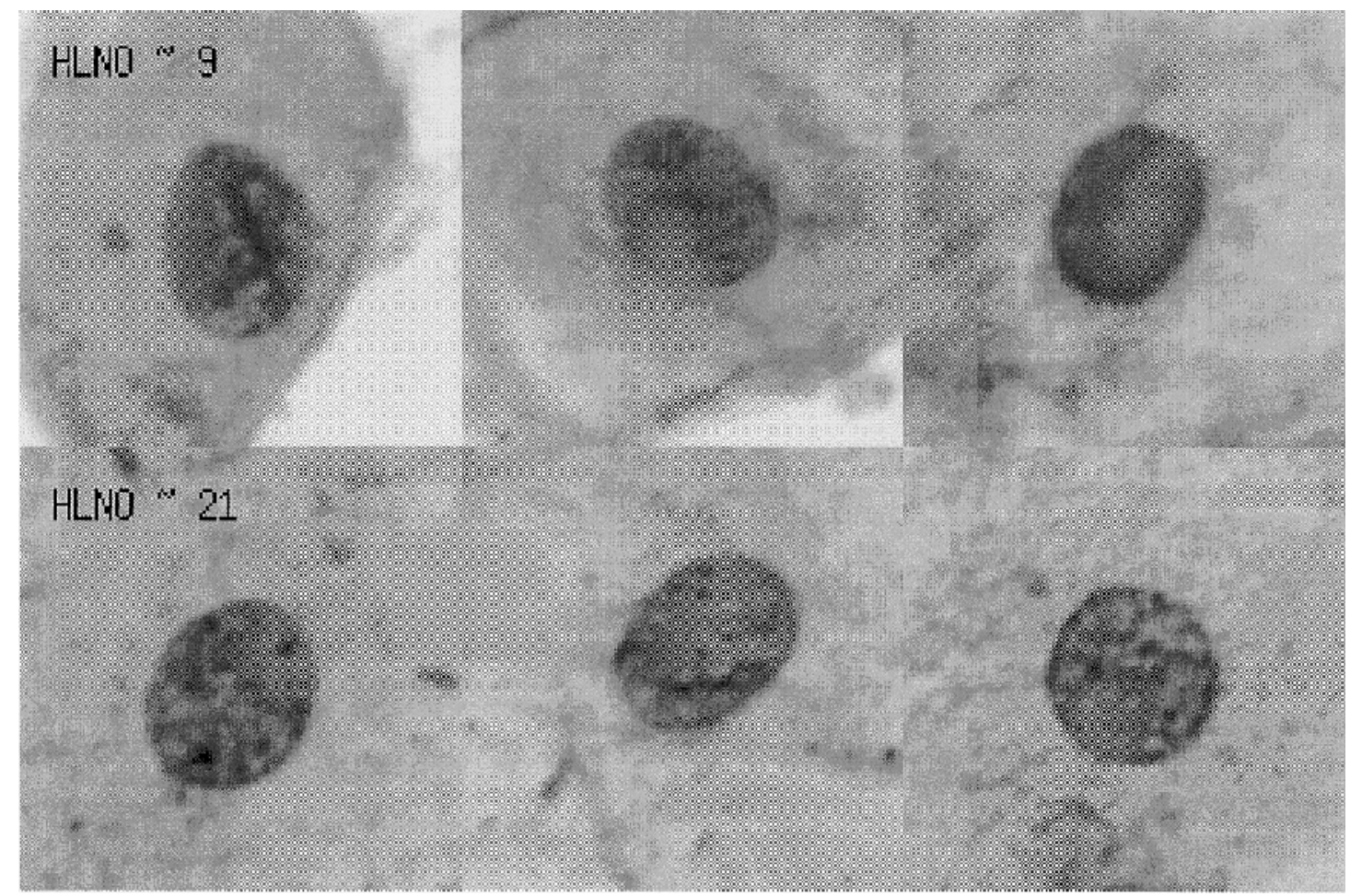

Fig. 5. Nuclei with low (9.0) and high (21.0) values of HLNO.

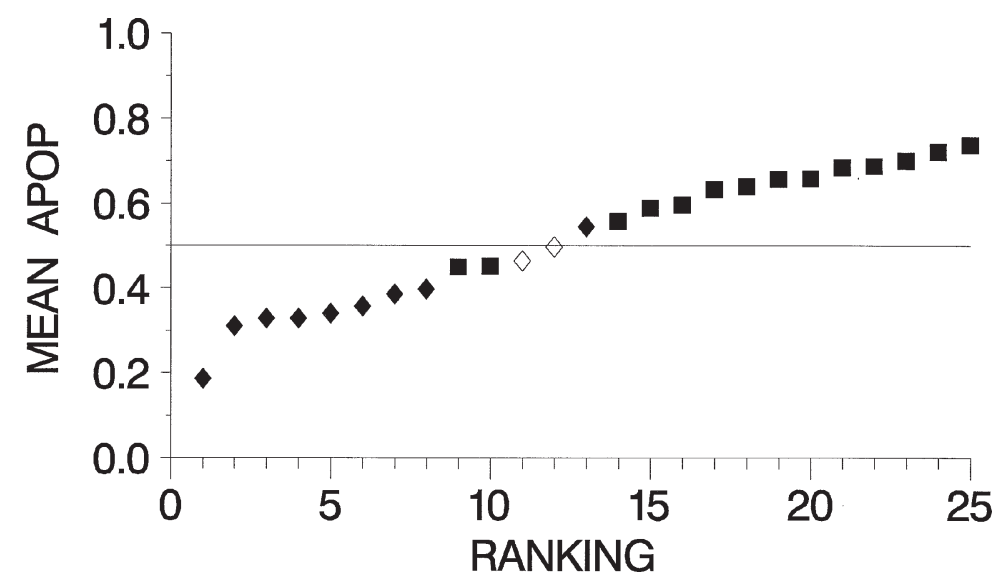

Fig. 6. Cell and specimen classification results of cases sampled in 1987. non-progression, open symbols: unclear decisions.) 
values of HLNO. The cell classification was $71.9 \%$ for the non-progression group and $71.1 \%$ for the progression group. 8/11 specimens of the non-progression group and 12/14 of the progression group were correctly classified with two unclear cases and one false decision in the non-progression group and two false decisions in the progression group (Fig. 6). In case of no unclear decisions both cases were correctly classified $(p<0.001)$.

\subsection{Discrimination of patients with non-progression and progression within five years at time of diagnosis (1989)}

Using the subset of chromatin features, correct cell classification rates of $65.3 \%$ for the nonprogression group and $74.6 \%$ for the progression group were achieved (Table 3). The best feature was once again NC9 followed by RGM2. The subsequent specimen classification resulted in 9/11 correct non-progression decisions and 11/14 correct progression decisions. Three cases of the progression group were unclear and two cases of the non-progression group were falsely classified (Fig. 7). In case of no unclear decisions two of the cases were correctly classified and one falsely $(p<0.0001)$.

Table 3

Cell and specimen classification results for 1989

\begin{tabular}{lccccc}
\hline & Non-progression & Progression & Total & $\%$ correct & \\
Non-progression & 874 & 464 & 1338 & 65.3 & \\
Progression & 355 & 1045 & 1400 & 74.6 & \\
& Non-progression & Progression & Unclear & Total & $\%$ correct \\
Non-progression & 9 & 2 & - & 11 & 78.6 \\
Progression & - & 11 & 3 & 14 & 92.9 \\
& Non-progression & Progression & Total & $\%$ correct & \\
Non-progression & 9 & 2 & 11 & 81.8 & \\
Progression & - & 14 & 14 & 100.0 & \\
\hline
\end{tabular}

$p<0.0001$.

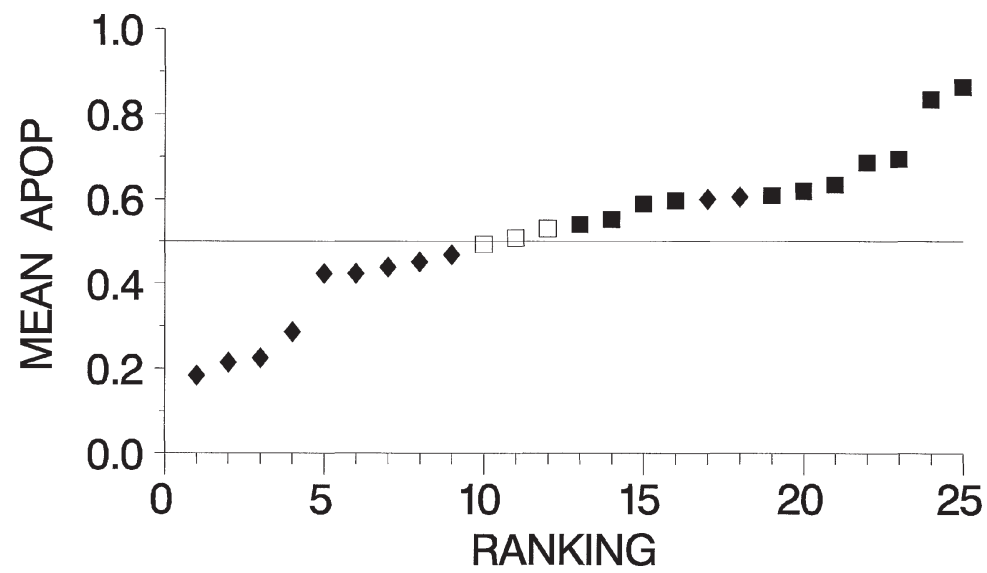

Fig. 7. Cell and specimen classification results of cases sampled in 1989. non-progression, open symbols: unclear decisions.) 


\section{Discussion}

Cancer chemoprevention is being taken seriously in recent years. Normally at present, the rate of cancer incidence reduction is used as the endpoint of clinical trial in the field of cancer chemoprevention. However the barriers to the development of chemoprevention is the unacceptable cost, long duration and large scale of effort. Therefore, it is urgent that this endpoint be replaced by the Surrogate Endpoint Biomarker (SEB) that occurs early during neoplastic development and which is precise enough to achieve adequate statistical study power. Boone et al. believed that the use of morphometric parameters through computer-assisted image analysis can be used to predict the progression and regression of precancerous lesions [1]. Palcic concluded from a cervix chemoprevention study that nuclear texture measurements of MACs can be used as a SEB [30]. In recent study, Herlin et al. used DNA ploidy abnormalities to classify esophageal intraepithelial neoplasia and invasive carcinoma [12]. Their results showed that the parameters were unable to discriminate one from the other. Meanwhile two other studies [39,44], using texture features as MAC, led to a possible discrimination of dysplasia and carcinoma of cervix, as well as in situ and invasive breast carcinoma.

Our results given above show that it is possible to discriminate esophageal severe dysplasia patients with non-progression and progression to carcinoma within a few years. In spite of differences in preparation and staining conditions which only permitted the pooling of specimens according to different years, the classification results have been surprisingly good with correct decisions of about $80 \%$. None of the patients of the progression group was classified as non-progression (1989) which should certainly be avoided. False positive decisions can be accepted if the subsequent treatments are not too aggressive.

The difference in significance of the selected features in certain classification steps were caused by the staining and preparation conditions. Nevertheless chromatin features extracted by high resolution image analysis were found to give significant additional information to the clinicians, about patients who will have a progress to cancer with a certain probability. In the future it may be possible that the pathologist can learn to differentiate patients with progression and non-progression, by means of textural features measured from normal appearing intermediate cells (Figs 2-4). Then according to the evaluated probability, an individual's treatment planning (operation, chemoprevention or reexaminations in shorter periods of time etc.) for the patient may be applied. Above all, it seems that features obtained from high resolution image analysis can be applicable as a marker of subtle changes in malignancy. Further, these features might be used as SEBs in cancer chemoprevention clinical trials.

Due to these surprisingly good results, a further prospective study using standardized air dried preparations and staining conditions was started in the meantime. Thus it should be possible to find that subset of chromatin features which is highly correlated with the prognosis of the dysplasia patients. Other questions such as the problem of which period the cancer actually occurs or the aggressivity of the tumour could be addressed.

\section{References}

[1] C.W. Boone and G.J. Kelloff, Development of surrogate endpoint biomarkers for clinical trials of cancer chemoprevention agents: relationships to fundamental properties of preinvasive (intraepithelial) neoplasia, Journal of Cellular Biochemistry Supplement 19 (1994), 10-22.

[2] G. Burger, M. Aubele, B. Clasen, U. Jütting, P. Gais and K. Rodenacker, Malignancy associated changes in squamous epithelium of the head and neck region, Analyt. Cell. Pathol. 7 (1994), 181-193. 
[3] G. Burger, U. Jütting and K. Rodenacker, Changes in benign cell populations in cases of cervical cancer and its precursors, Analyt. Quant. Cytol. 3 (1981), 261-271.

[4] G. Burger and U. Jütting, Specimen classification in cytometry: an intercomparison of various means of decision making, in: Pattern Recognition in Practice II, E.S. Gelsema, L.N. Kanal, eds, North-Holland Publ., Amsterdam, 1985, pp. 509-519.

[5] W.D. Dupont, F.F. Parl, W.H. Hartmann, L.A. Brinton, A.C. Winfield, J.A. Worrel, P.A. Schuyler and W.D. Plummer, Breast cancer risk associated with proliferative breast disease and atypical hyperplasia, Cancer 71 (1993), 1258-1265.

[6] B.S. Erler, L. Hsu, H.M. Truong, L.M. Petrovic, S.S. Kim, M.H. Huh, L.D. Ferrell, S.N. Thung, S.A. Geller and A.M. Marchevsky, Image analysis and diagnostic classification of hepatocellular carcinoma using neural networks and multivariate discriminant functions, Lab. Invest. 71 (1994), 446-451.

[7] R.R. Finch, A classification of nuclear aberration in relation to Malignancy Associated Changes (MAC), Acta Cytol. 16 (1971), 553-558.

[8] P. Gais and G. Burger, Geräte zur quantitativen Mikroskopie, in: Morphometrie in der Zyto- und Histopathologie, G. Burger, M. Oberholzer and W. Gössner, Hrg., Springer-Verlag, New York, 1988, pp. 282-294.

[9] F. Gilles, A. Gentile, V. Ledoussal and E. Kahn, Use of texture parameters in the classification of soft tissue tumors, Analyt. Quant. Cytol. Histol. 16 (1994), 315-320.

[10] M. Guillard, A. Doudkine, D. Garner, C. MacAulay and B. Palcic, Malignancy associated changes in cervical smears: systematic changes in cytometric features with grade of dysplasia, Analyt. Cell. Pathol. 9 (1995), 191-204.

[11] G. Haroske, St. Bergander, R. König and W. Meyer, Application of malignancy-associated changes in the cervical epithelium in a hierarchical classification concept, Analyt. Cell. Pathol. 2 (1990), 189-198.

[12] P. Herlin, F. Duigou, E. Masson, J. Marney, J.L. Liu, Henryarma, D. Bloyet, J.C. Mandard and A.M. Mandard, DNA cytometric abnormalities in human esophageal squamous intraepithelial and invasive carcinoma, Analyt. Quant. Cytol. Histol. 17 (1995), 8-14.

[13] H. Klawe and J. Rowinski, Malignancy associated changes (MAC) in cells of buccal smears detected by objective image analysis, Acta Cytol. 18 (1974), 30-33.

[14] M.D. Lagios, F.R. Margolin, P.R. Westdahl and M.R. Ross, Mammographically detected duct carcinoma in situ. Frequency of local recurrence following tylectomy and prognostic effect of nuclear grade on local recurrence, Cancer 63 (1989), 618-624.

[15] S. Lam, C. MacAulay and B. Palcic, Detection and localization of early lung cancer by image techniques, Chest 103 (1993), 12S-14S.

[16] P.Z. Lin, J.S. Zhang, Z.P. Rong, R. Han, S.P. Xu, R.Q. Gao and S.G. Cao, Second line prevention from esophageal cancer inhibitory therapy to block precancerous lesions, Chinese Journal of Cancer Research 1 (1988), 37-46.

[17] P.Z. Lin, J.S. Zhang, Z.P. Rong, R. Han, S.P. Xu, R.Q. Gao, Z.W. Ding, J.X. Wang, H.J. Feng and S.G. Cao, Studies on medicamentous inhibitory therapy for esophageal precancerous lesions 3- and 5-year inhibitory effects of Antitumor-B, Retinamide and Riboflavin, in: Proc. CAMS and PUMC 5 (1990), pp. 121-129.

[18] P.Z. Lin, Introduction of research works in esophageal precancerous lesions (dysplasia of esophageal epithelium), Chinese Journal of Cancer 5 (1983), 391.

[19] S.J. London, J.L. Connelly, S.J. Schnitt and G.A. Colditz, A prospective study of benign breast disease and the risk of breast cancer, JAMA 267 (1992), 941-944.

[20] C. MacAulay and B. Palcic, Fractal texture features based on optical density surface area: Use in image analysis of cervical cells, Analyt. Quant. Cytol. Histol. 12 (1990), 394-398.

[21] J. Miller, D.J. Horsfall, J.M. Skinner, D.M. Rao, A.S.Y. Leong and V.R. Marshall, Nuclear shape and prognosis following orchiectomy in stage D-2 prostate cancer, Prostate 24 (1994), 306-312.

[22] H.E. Nieburgs, B.E. Herman and H. Reisman, Buccal cell changes in patients with malignant tumors, Lab. Invest. 2 (1962), 80-88.

[23] H.E. Nieburgs, A.D. Parets, V. Perez and C. Boudreau, Cellular changes in liver tissue adjacent and distant to malignant tumors, Arch. Pathol. 80 (1965), 262-272.

[24] H.E. Nieburgs, F.G. Zak, D.C. Allen, H. Reisman and T. Clardy, Systemic cellular changes in material from human and animal tissues in presence of tumors, in: Proc. Int. Soc. Cytol. Council, Seventh Annual Meeting, 1959, pp. 137-144.

[25] H.E. Nieburgs, Cutaneous malignancy associated changes in cancer of the skin and other sites. Biology-diagnosismanagement, in: Cancer of the Skin, Vol. 1, R. Andrade, S.L. Gumport, G.L. Popkin and T.D. Rees, eds, W.B. Saunders, Philadelphia, 1976.

[26] H.E. Nieburgs, Recent progress in the interpretation of Malignancy Associated Changes (MAC), Acta Cytol. 12 (1968), $445-453$.

[27] G.R. Odgen, J.G. Cowpe and M.W. Green, The effect of distant malignancy upon quantitative cytologic assessment of normal oral mucosa, Cancer $\mathbf{6 5}$ (1990), 477-480.

[28] B. van Oppen, A. Meisels and S. St-Pierre, Malignancy associated changes in sputum: a correlated study of 315 patients, Acta Cytol. 19 (1975), 573-576. 
[29] B. Palcic, B. Susnik, D. Garner and I. Olivotto, Quantitative evaluation of malignant potential of early breast cancer using high resolution image cytometry, Journal of Cellular Biochemistry 17G (Suppl.) (1993), 107-113.

[30] B. Palcic, Nuclear texture: Can it be used as a surrogate endpoint biomarker?, Journal of Cellular Biochemistry 19 (Suppl.) (1994), 40-46.

[31] B. Palcic and C. MacAulay, Malignancy associated changes: Can they be employed clinically?, in: Compendium on the Computerized Cytology and Histology Laboratory, G.L. Wied and D.L. Rosenthal, eds, Tutorial of Chicago, II, USA, 1994, pp. 157-165.

[32] A.K. Reith, S. Reichborn-Kjennerud, M. Aubele, U. Jütting, P. Gais and G. Burger, Biological monitoring of chemical exposure in nickel workers by image cytometry (ICM) of nasal smears, Analyt. Cell. Pathol. 6 (1994), 9-21.

[33] F. Rilke and S. Pilotti, Data on malignancy associated changes in oral and bronchial epithelial cells in sputum specimens: their possible utilization for identification and follow up of high risk groups, Cancer Chemother. (Rep. Part 3) 4 (1973), 73-78.

[34] K. Rodenacker, Featuring of cellular objects, in: Clinical Cytometry and Histometry (Rep. Part 3), G. Burger, J.S. Ploem and K. Goerttler, eds, Vol. 4, 1973, pp. 73-78.

[35] K. Rodenacker, Invariance of textural features in image cytometry under variantion of size and pixel magnitude, Analyt. Cell. Pathol. 8 (1995), 117-133.

[36] J. Schmidt, M. Aubele, U. Jütting, K. Rodenacker, A. Luz, V. Erfle and G. Burger, Computer-assisted imaging cytometry of nuclear chromatin reveals bone tumor virus infection and neoplastic transformation of adherent osteoblastlike cells, Biochem. Biophys. Res. Commun. 164 (1989), 728-735.

[37] D. Seigneurin, J. Louis and M.C. Villoud, The value of DNA image cytometry for the cytological diagnosis of well-differentiated breast carcinomas and benign lesions, Analyt. Cell. Pathol. 7 (1994), 115-125.

[38] B. Susnik, A. Worth, J. Leriche and B. Palcic, Malignancy-associated changes in the breast - Changes in chromatin distribution in epithelial cells in normal-appearing tissure adjacent to carcinoma, Analyt. Quant. Cytol. Histol. 17 (1995), 62-68.

[39] B. Susnik, Quantitative nuclear features in the prognosis of benign breast disease and ductal carcinoma in situ. $\mathrm{PhD}$ Thesis, University of British Columbia, January 28, 1994.

[40] R. Vanvelthoven, M. Petein, A. Zlotta, W.J. Oosterlinck, A. Vandermeijden, C. Zandona, H. Roels, J.L. Pasteels, C. Schulman and R. Kiss, Computer-assisted chromatin texture characterization of Feulgen-stained nuclei in a series of 331 transitional bladder cell carcinomas, Journal of Pathology 173 (1994), 235-242.

[41] G.L. Wied, P.H. Bartels, M. Bibbo and J.J. Sychra, Cytophotometric markers for uterine cancer in intermediate cells, Analyt. Quant. Cytol. 2 (1980), 257-263.

[42] G.L. Wied, M. Bibbo, F.T. Pishotta and P.H. Bartels, Intermediate cell markers for malignancy, Analyt. Quant. Cytol. 6 (1984), 243-246.

[43] W.H. Wolberg, W.N. Street and O.L. Mangasarian, Machine learning techniques to diagnose breast cancer from image-processed nuclear features of fine needle aspirates, Cancer Letters 77 (1994), 163-171.

[44] G. Wolf, M. Bell and H. Guski, Chromatin structure analysis based on a hierarchic texture model, Analyt. Quant. Cytol. Histol. 17 (1995), 25-34

[45] I.T. Young, P.W. Verbeek and B.H. Mayall, Characterization of chromatin distribution in cell nuclei, Cytometry 7 (1986), 467-474.

\section{Appendix}

Feature description:

HETERO Heterogeneity of chromatin (Mayall/Young) [45]

HLNO Number of dark particles, equals to the number of dark regions

NC9 Sum entropy of flat texture co-occurrence matrix

MFRANG Range of local multifractal dimension

RGM2 SD of topological gradient

RL4 Runlength non-uniformity of extinction image

RFM1 Ratio of mean optical density of the rim and the nucleus

NC11 Difference entropy of flat texture co-occurrence matrix

NR5 Runlength percentage of flat texture runlength matrix

MFM3 Skewness of local multifractal dimension 


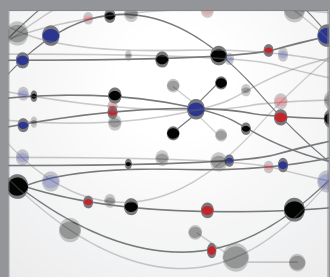

The Scientific World Journal
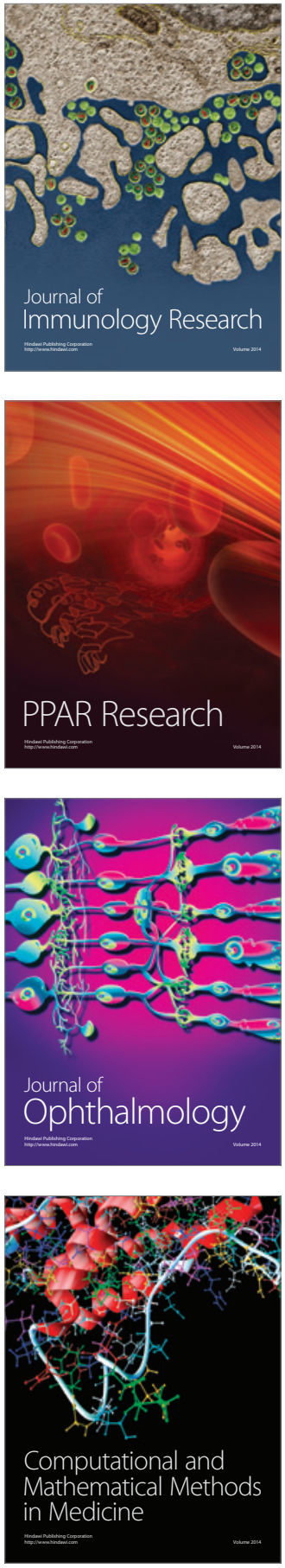

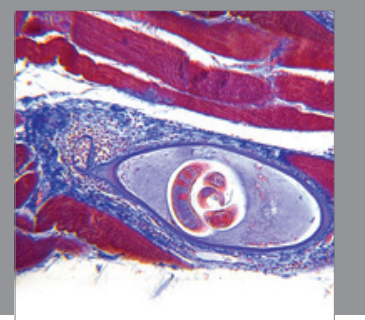

Gastroenterology

Research and Practice
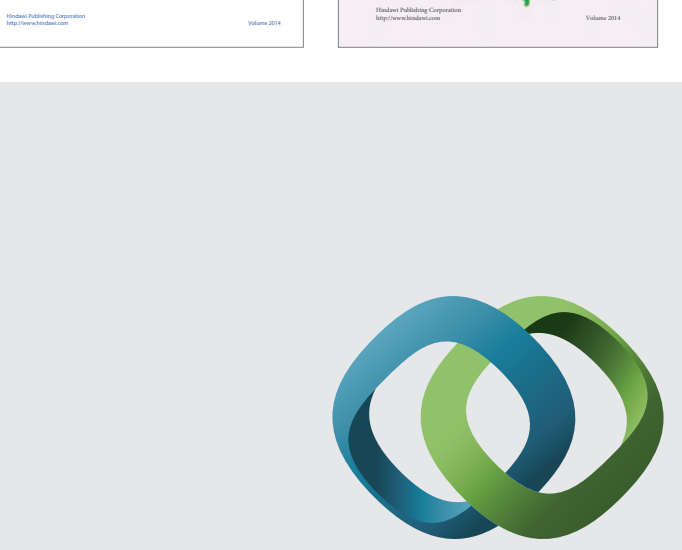

\section{Hindawi}

Submit your manuscripts at

http://www.hindawi.com
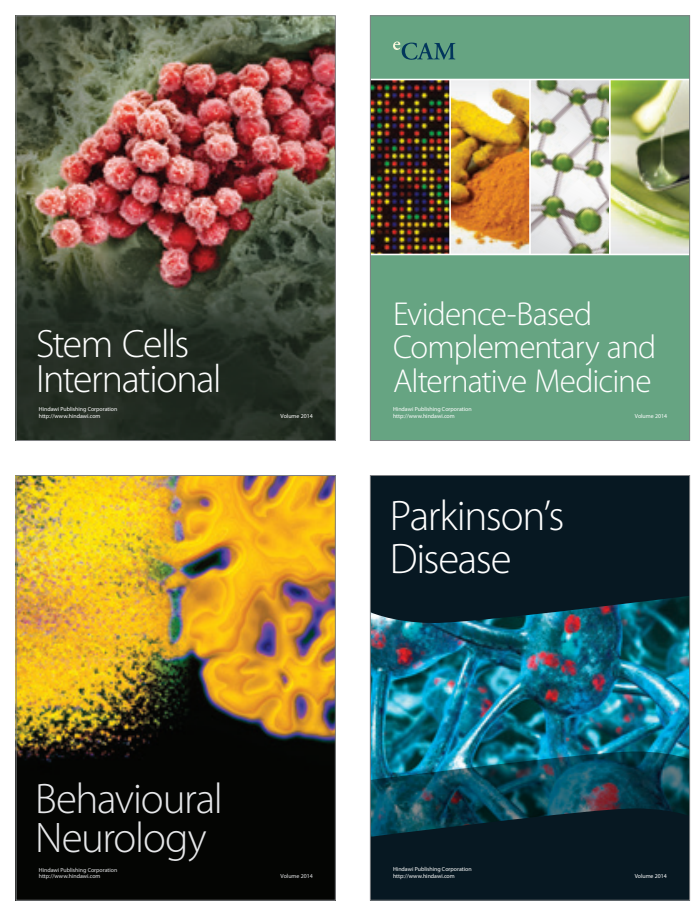

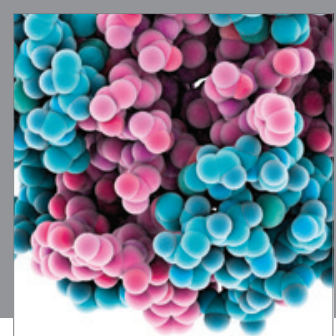

Journal of
Diabetes Research

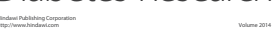

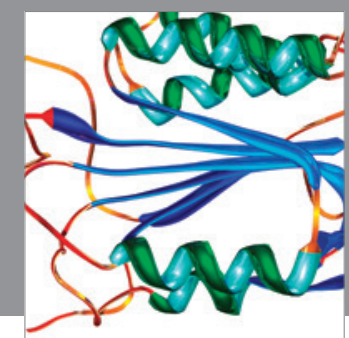

Disease Markers
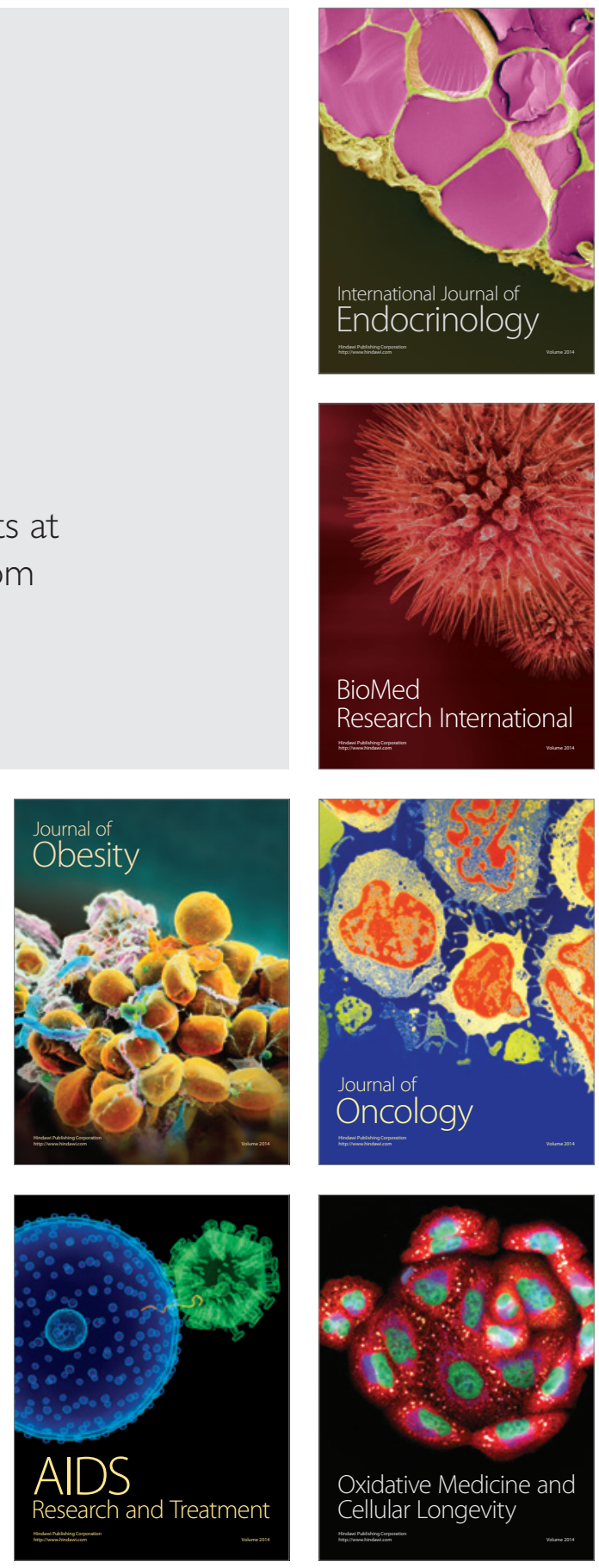